

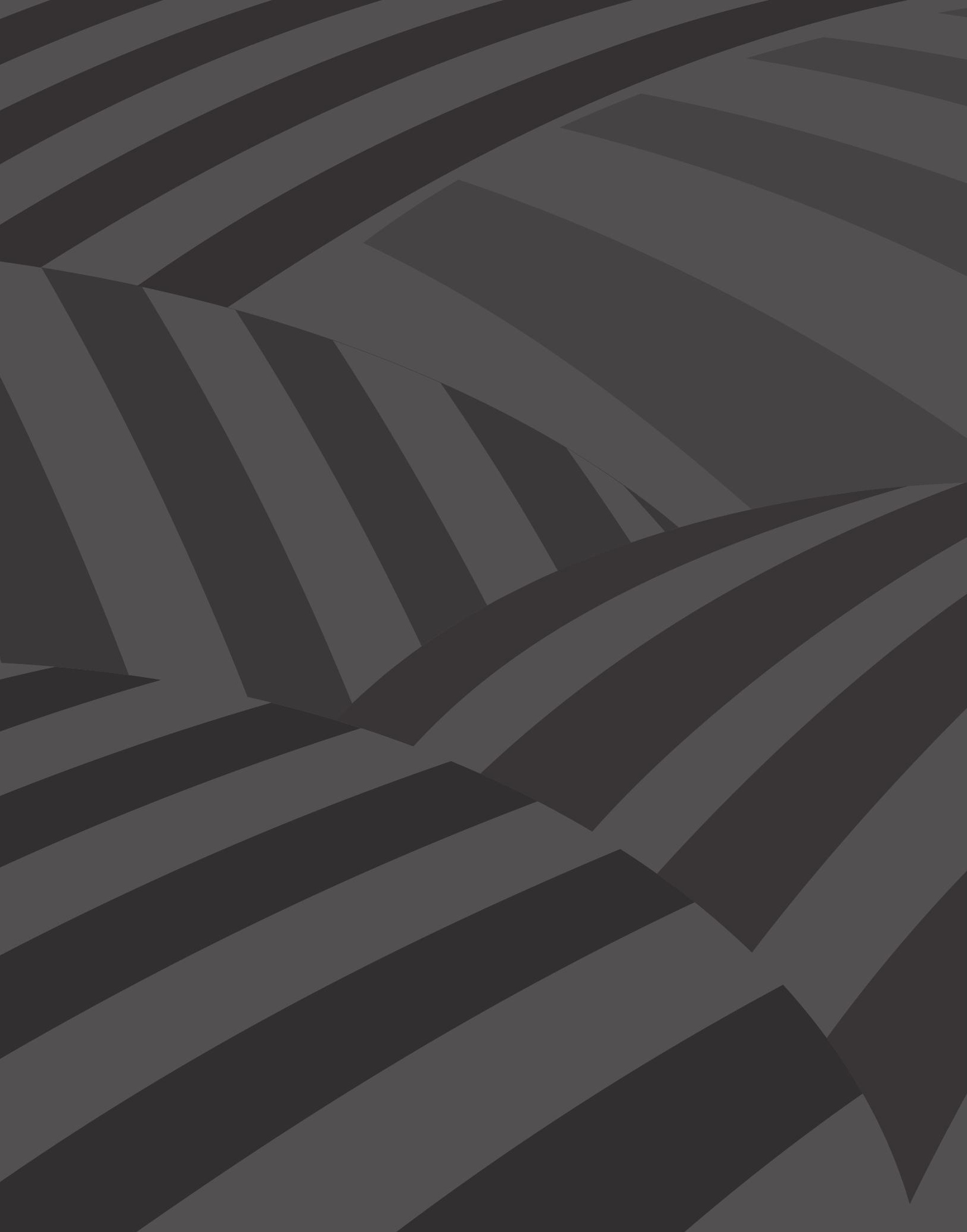




\section{Caldono, territorio para la paz. Tensiones en el primer año de implementación del acuerdo final en los resguardos indígenas que acogieron excombatientes de las FARC}

Por Alejandra Erazo Gómez* y Luisa Espitia Pérez ${ }^{* *}$

Resumen: el artículo presenta un análisis sobre las tensiones surgidas en el municipio de Caldono (Cauca) durante el primer año de implementación del acuerdo entre el Gobierno nacional y las FARC-EP. Este municipio tiene la particularidad de ser el único donde se estableció una Zona Veredal Transitoria de Normalización en territorio étnico, en las jurisdicciones de los resguardos indígenas de Pueblo Nuevo y San Lorenzo de Caldono. Las tensiones identificadas por el Instituto de Estudios Interculturales de la Pontificia Universidad Javeriana de Cali en ambos resguardos están relacionadas con el territorio, la dinámica económica y los procesos de reincorporación de excombatientes de las FARC-EP en el municipio. Su identificación genera reflexiones importantes para el proceso de implementación del acuerdo.

Palabras clave: FARC-EP, reincorporación, resguardos indígenas, tensiones territoriales.

\section{Caldono, Peace Territory. Tensions in the Indigenous Reservations Hosting former FARC members during the First Year of Implementation of the Final Agreement}

Abstract: The article presents an analysis of the tensions that arose in the municipality of Caldono (Cauca, Colombia) during the first year of implementation of the Peace Agreement between the national government and the FARC-EP guerrilla. This municipality is the only one in Colombia where a Transitory Normalization Zone was established in ethnic territory, property of two indigenous reserves: Pueblo Nuevo and San Lorenzo of Caldono. The tensions, identified by the Institute of Intercultural Studies, are associated to land property, economic issues and the reincorporation process into civilian lives of FARC-EP ex-combatants in the municipality. The identification of the

* Investigadora del Instituto de Estudios Interculturales de la Universidad Javeriana, seccional Cali. Antropóloga de la Universidad Icesi. Correo electrónico: alejandra. erazo@javerianacali.edu.co

** Investigadora del Instituto de Estudios Interculturales de la Universidad Javeriana, seccional Cali. Socióloga de la Universidad Nacional de Colombia. Correo electrónico: luisa.espitia@javerianacali.edu.co 
aforementioned tensions enables important insights for the ongoing implementation process of the Peace Agreement in Colombia.

Keywords: FARC-EP, reincorporation process, indigenous reserves, social tensions associated to land.

Cómo citar este artículo: Erazo, Alejandra y Espitia, Luisa (2018). Caldono, territorios para la paz. Tensiones en el primer año de implementación del acuerdo final en los resguardos indígenas que acogieron excombatientes de las FARC. Revista Controversia, 210, 45-83.

Fecha de recepción: 28 de febrero del 2018.

Fecha de aprobación: 7 de mayo del 2018.

\section{Introducción}

$\mathrm{E}$

n el marco de la implementación del acuerdo firmado entre las FARC-EP y el Gobierno nacional en 2016 para la terminación del conflicto y la construcción de una paz estable y duradera, se estableció en el municipio de Caldono, departamento del Cauca, una de las Zonas Veredales Transitorias de Normalización (ZVTN) en las que se agruparon los y las excombatientes del grupo subversivo para llevar a cabo el proceso de dejación de armas. Esta fue la única ZvTN localizada dentro de un territorio indígena. Su ubicación responde a múltiples factores, entre los que se encuentran la presencia histórica de esta guerrilla en la región, la profunda afectación del territorio ancestral por el conflicto armado y la reivindicación histórica del movimiento indígena por la construcción de paz. No obstante, la decisión de las comunidades indígenas de Caldono de abrir sus puertas para acoger a los y las excombatientes durante la dejación de armas derivó en conflictos territoriales frente al proceso de instalación de la ZVTN y tensiones relacionadas con la organización política indígena y su ejercicio de autonomía, así como otras vinculadas al proceso de reincorporación social, política y económica de los excombatientes de las FARC.

No desconocemos que estos conflictos hayan surgido de un trasfondo histórico complejo, que excede el periodo de implementación del 
acuerdo de paz, y hunden sus raíces en la disputa por la tierra en el Cauca, las reivindicaciones de los pueblos indígenas en el departamento y la presencia continua de actores armados en la región de Caldono. Aun cuando algunos de estos temas se delinean a lo largo del artículo, el enfoque del texto supone la presentación de las tensiones y conflictos territoriales en el estado en que se encontraban en 2017.

La información presentada fue recopilada a partir del trabajo de acompañamiento al Resguardo de Pueblo Nuevo, ubicado en la zona sur del municipio de Caldono, que el Instituto de Estudios Interculturales ha hecho desde 2015. Este proceso incluye el estudio exploratorio "Paz territorial y conflictos en Zonas Veredales Transitorias de Normalización para excombatientes de las FARC”, apoyado por el Instituto ColomboAlemán para la Paz (Capaz) ${ }^{1}$, en el que también fueron consultadas las autoridades del Resguardo San Lorenzo de Caldono y las comunidades campesinas de la vereda La Venta.

El texto se organiza en cinco partes. La primera es una contextualización del municipio a través de una breve presentación de su historia y de las dinámicas territoriales, productivas y organizativas. La segunda parte se centra en la descripción de los aspectos relevantes que influyeron en la decisión de instalar la ZVTN en el municipio y de los efectos de su llegada al territorio. En un tercer apartado se abordan las tensiones que este hecho conlleva en lo político con relación al movimiento indígena y a los ejercicios de autonomía en el territorio. En un cuarto punto se hace referencia a otras tensiones relacionadas con la reincorporación de excombatientes, lo que incluye preguntas alrededor de los recursos compartidos, la situación de seguridad en el municipio y las apuestas económicas que las mujeres y hombres de las FARC planean desarrollar en el territorio. Por último, se reconocen los gestos de reconciliación que

1 El estudio contó con la participación de investigadoras de la Universidad del Valle, el Instituto de Estudios Interculturales de la Universidad Javeriana de Cali, la Universidad Cooperativa de Colombia, sede Pasto, y la organización no gubernamental Cetec. La participación del Instituto se enfocó en el municipio de Caldono. 
han tenido lugar en la zona y se propone un diálogo intercultural como apuesta para alcanzar consensos en procesos complejos como el que expone el documento.

\section{Dinámicas del municipio de Caldono}

El municipio de Caldono está ubicado en la vertiente occidental de la cordillera central y se extiende sobre un área de 35526 hectáreas que agrupan 86 veredas y cuatro corregimientos: Cerro Alto, Siberia, Pescador y Pital. Las proyecciones del Censo de 2005 estiman que para 2018 la población asciende a 33910 personas, lo que representa el 2,4 \% de la población del departamento del Cauca (DANE, 2005). La distribución de la población es predominantemente rural: menos del $5 \%$ se concentra en la cabecera. A su vez, el 65,6\% se reconoce a sí mismo como indígena y se ubica mayormente al oriente del municipio.

En este municipio están los resguardos de La Laguna Siberia, constituido en 1994, y Páez de las Mercedes, constituido en 2003, que en conjunto suman aproximadamente 320 ha. Igualmente, cuenta con cuatro resguardos con títulos de origen colonial: San Lorenzo de Caldono, Pueblo Nuevo, La Aguada y Pioyá, para un total de seis resguardos pertenecientes a la comunidad nasa, que alcanzan una extensión aproximada de 21000 ha y conforman el Territorio Ancestral Sa'th Tama Kiwe, que alude al "territorio del cacique Juan Tama” o "tierra del gran cacique". En el corregimiento de Siberia también se encuentra el cabildo misak La Laguna, que en 2010 estaba conformado por 360 familias que habitaban 139 ha (Bonilla, 2012) y actualmente no cuenta con titulación colectiva.

Caldono se caracteriza por una economía basada en agricultura familiar y comunitaria y por la marcada diferencia entre las dinámicas productivas de su zona alta, tradicionalmente indígena (nasa), y su zona plana, principalmente campesina. La zona alta no cuenta con la totalidad del terreno para la agricultura, pues allí se encuentran las áreas de protección, que suman el 14,5 \% de la extensión del municipio (5169 ha) y en su 
totalidad se ubican en territorio de los resguardos coloniales (PDM, 20162019). Los cabildos son los encargados de velar por el cuidado de estas áreas, que en sus planes de vida son comprendidas como espacios de vida. En el área restante, los principales productos agrícolas sembrados son el fique, el café, el fríjol, los frutos de clima frío como la mora y el tomate de árbol; predominan las huertas de pancoger.

Los datos de producción del Censo Nacional Agropecuario (2014) para estos territorios contrastan con el indicador de producción para el autoconsumo, lo que permite concluir que existe una baja comercialización de productos. La comercialización se da principalmente en lo local, en los mercados de los centros poblados de los resguardos cercanos, producto de la precaria infraestructura productiva en la zona alta del municipio (vías angostas y sin pavimentar, falta de centros de acopio y de transformación de productos) y la baja diversificación de cultivos.

El principal cultivo en la zona es el fique. Según Rojas (1993), en la década de los sesenta, se consolidó este cultivo a partir de un aumento de demanda industrial por parte de las compañías productoras de empaques; sin embargo, en las siguientes dos décadas sobrevino una crisis de su comercialización por el cambio a fibras sintéticas para la elaboración de empaques; desde entonces, este cultivo no ha sido sustituido en su totalidad. Por otro lado, la zona plana cuenta con cultivos de café, piña, caña panelera, fríjol, entre otros, así como también con algunos espacios para la transformación de productos como trapiches y rayanderías.

Los cabildos del municipio están organizados con fines principalmente administrativos en la Asociación de Cabildos de Caldono Ukawe'sx Nasa Çxhab. Esta organización no reemplaza a la autoridad tradicional que reside en los cabildos, principalmente en el caso de los gobernadores indígenas. La existencia de la asociación evidencia la lucha de las comunidades indígenas del municipio por su autonomía e incidencia en el nivel departamental y regional, lucha que han dado incluso dentro del 
movimiento indígena aun cuando no hagan parte de la Asociación de Cabildos Indígenas del Norte del Cauca, debido a diferencias políticas y en las dinámicas territoriales de la subregión. Sin embargo, sí pertenecen a uno de los diez proyectos zonales del Consejo Regional Indígena del Cauca (CRIC).

Los seis resguardos cuentan con guardias indígenas encargadas del control territorial, conformadas por comuneras y comuneros que son seleccionados por las autoridades del cabildo para un período de uno a dos años. La guardia indígena facilita el ejercicio de justicia propia y contribuye a la construcción de autonomía. Su organización propone "una alternativa a los modelos de control territorial de los actores armados insurgentes, paraestatales y estatales, desde el reconocimiento de la autoridad tradicional y no desde el uso de la fuerza" (Caviedes, 2007, p. 57). La guardia es el resultado de años de organización para la recuperación de territorios despojados durante la época republicana y, más recientemente, para el control del territorio recuperado. La sistematización realizada por el Centro de Cooperación al Indígena Cecoin (2007) señala que la guardia existe en su forma actual por lo menos desde 1960 y, en la década de 1990, se formaliza como una institución con presencia permanente en la comunidad. El primer coordinador de la guardia fue nombrado en el resguardo de San Lorenzo de Caldono. En el municipio, la guardia ha tenido incidencia en temas de delincuencia común, promoción de la participación de jóvenes en la organización, interlocución con fuerza pública, mediación con grupos insurgentes para la liberación de personas secuestradas y acompañamiento a movilizaciones por la defensa y exigencia de derechos (Caviedes, 2007).

Por otro lado, en el municipio hace presencia la Coordinación Nacional de Pueblos Indígenas (CONPI), un proceso adscrito nacionalmente al movimiento social y político Marcha Patriótica, que surge como propuesta alternativa a las organizaciones indígenas tradicionales como ACIN y CRIC, y señala diferencias en cuanto a la estructura organizativa, las agendas y los repertorios de acción de dichas organizaciones. La 
CONPI agrupa a la Asociación Regional Indígena por la Defensa de la Soberanía Alimentaria (Arisa) y el movimiento Sin Tierra Nietos de Quintín Lame. Dichas organizaciones se agrupan alrededor de lo productivo y lo político y no se encuentran articuladas a la dirección de los cabildos y proyectos zonales. En esta dirección, se han constituido en una fuerza que se disputa asuntos importantes con los cabildos y el CRIC como las narrativas del conflicto armado y los repertorios de acción propios de la movilización.

El movimiento Sin Tierra Nietos de Quintín Lame hace presencia en los municipios de Santander de Quilichao, Caloto, Corinto, pero su mayor presencia organizativa está en Caldono. Dicha organización establece dentro de su repertorio político la recuperación de tierras y viene adelantado procesos productivos importantes en el municipio alrededor de la producción de café, frutales y producción agroecológica.

Por su parte, la Arisa es una asociación constituida legalmente en julio de 2009, con subdirectivas en Jambaló, Toribío, Cajibío, Suárez, Santander, Caldono, Silvia y Morales. Su objetivo apunta específicamente al fortalecimiento productivo. Actualmente, agrupa a gran parte de los productores de coca del municipio y viene avanzando en discusiones sobre la sustitución de cultivos de uso ilícito y la implementación del punto 4 del acuerdo de la Habana.

De manera complementaria, en el municipio hacen presencia varias organizaciones campesinas, entre las que se destacan la Asociación Nacional de Usuarios Campesinos (ANUC), la Asociación Campesina de Caldono (Asocal) y la Asociación Regional para el Desarrollo Campesino Nortecaucano (Ardecanc). Como se puede evidenciar, hay una fuerte presencia de movimientos sociales regionales y nacionales alrededor de la organización indígena y campesina, que han fortalecido lo productivo y organizativo en el territorio desde diferentes visiones políticas y han sido afectados por los enfrentamientos armados allí ocurridos. 
Figura 1. Resguardos indígenas y organizaciones sociales en Caldono.

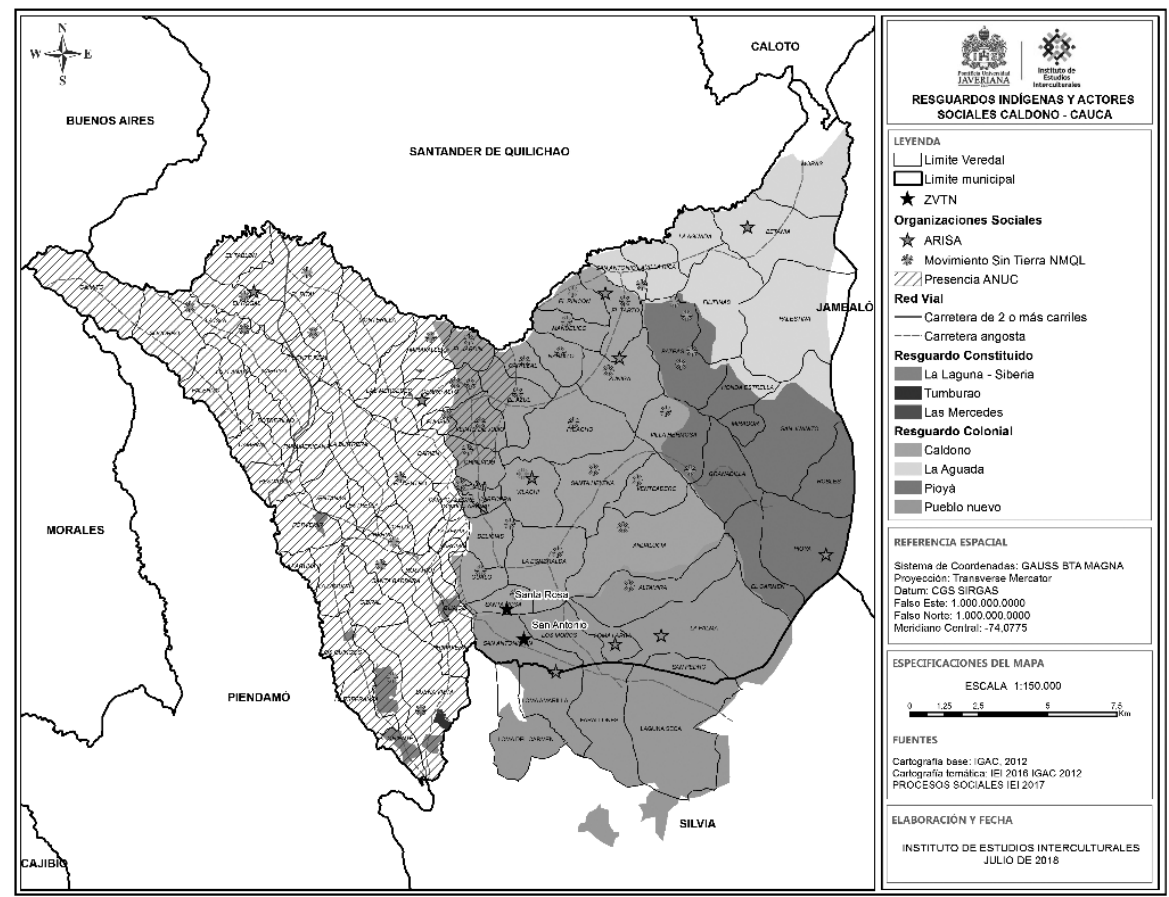

\section{Contexto histórico}

En Caldono, la configuración actual de la presencia indígena, mayoritariamente nasa, se remonta a las transformaciones que sufrió esta población a raíz de la invasión española. Según la investigación dirigida por José María Rojas (1993), el poblamiento de los paeces en Caldono responde a una migración de este grupo desde Tierradentro, donde se habían asentado en el siglo XIV, con centro político en La Plata, según señala Rappaport (2000). A la llegada de los españoles, los paeces combatieron y resistieron fuertemente sus incursiones durante un siglo; tras la irrupción española en tierras nasa en 1540 y su posterior expansión hasta el siglo XVIII, este pueblo se vio diezmado y forzado a desplazarse a partes más altas de la cordillera central. Después de la derrota, luego de 
dos siglos de enfrentamientos, los nasa del Cauca fueron incorporados como peones al sistema de haciendas en la Colonia.

Como estrategia para mantener a su población, transformaron su organización política a partir de "la consolidación del poder por parte de caciques cuyo dominio se legitimaba a través de las instituciones españolas e indígenas” (p. 75). Rappaport explica que con este objetivo se retomó el sistema de resguardo creado en la segunda mitad del siglo XVI por la corona española, el cual buscaba un mayor control sobre las poblaciones indígenas en América, incluido el pago de tributo, a cambio de que estas tuvieran un cierto grado de autonomía política y autogobierno. Los nasa se apropiaron de esta figura administrativa de manera que contribuyera a su defensa territorial. Así fue como en 1700, la Real Audiencia de Quito le otorgó a don Juan Tama de la Estrella el cacicazgo de Pitayó, ubicado en las laderas occidentales de la cordillera, que anexaba a Pitayó, Jambaló, Quichaya, Pueblo Nuevo y Caldono (Rojas, 1993). De esta manera, cinco cacicazgos paeces conformaron una unidad política que ejerció autonomía y logró conservar una tradición cultural en cabeza del cacique. Aquel territorio conformado por Tama es la base para los procesos de recuperación de tierra y la exigencia de reconocimiento estatal de la propiedad colectiva de los pueblos indígenas (Caviedes, 2007).

No obstante, en el periodo republicano "las comunidades indígenas se encontraron enfrentando un nuevo sistema político que les negaba la autonomía en favor de los intereses de la unidad nacional y de la expansión capitalista, que beneficiaba a la élite dominante" (Rappaport, 2000, p. 113). En este clima político, en el que interesaba la conformación de una nación mestiza, con una sola lengua y religión, el título de Juan Tama es declarado apócrifo en 1899. La élite mestiza proclamó el desmantelamiento del sistema de resguardo, con el objetivo de civilizar a los indígenas para que se articularan a la nación como ciudadanos, hispanohablantes, propietarios, asalariados, sin lealtad a otra autoridad que no fuera el Estado. A partir de aquel momento, se emprendieron 
acciones de desposesión e invasión de las tierras comunales de resguardo en el Cauca, que fueron desaceleradas por la Quintinada, en cabeza de Manuel Quintín Lame, durante las primeras décadas del siglo Xx.

Para la década de 1940 se profundizó la privatización de las tierras de resguardo y se expandieron las zonas de colonización sobre estas tierras. La violencia política de la década siguiente resultó en la disolución de los resguardos de San Lorenzo de Caldono y La Aguada, los cuales se parcelaron y vendieron como propiedad individual. Sus tierras fueron invadidas por colonos blancos, mestizos e incluso algunos indígenas que adquirieron títulos de propiedad. La autoridad indígena fue disuelta; Pioyá fue el

único resguardo que logró conservar intacto su territorio [...], fundamentalmente, por haber conservado la autoridad indígena del Cabildo. Y al haber opuesto resistencia a las distintas incursiones sobre su territorio, Pioyá logró sortear toda la violencia política y mantener la condición de polo de poder indígena. (Rojas, 1993, p. 103)

Fue hasta la década de 1970, con la creación del CRIC en 1971, cuando inicia el periodo de "reconstrucción del antiguo territorio del resguardo mediante la disolución de haciendas de terraje y ganadera” (p. 26). Rappaport (2000) plantea que la influencia del CRIC se asentó con más fuerza en el lado occidental de la cordillera central, puesto que allí persistía el terraje, las tierras de resguardo y había poca influencia de la Iglesia católica. En Caldono, la recuperación tuvo epicentro en la vereda San Antonio, del resguardo La Aguada, época en la cual fueron reconstruidos ambos resguardos extintos. En los años siguientes, con el avance de las guerrillas en el Cauca, los indígenas lucharon por mantener su autoridad en los resguardos. El polo indígena en Caldono se unió a las redes políticas de los partidos tradicionales, particularmente al Partido Liberal. Rojas (1993) señala que en años pasados el Partido Conservador administró el municipio de forma que concentró las inversiones en la parte baja donde predomina la presencia campesina, de manera que negó la intervención en la parte alta de población 
mayoritariamente indígena. Así mismo, ilustra cómo el poder que llegó a tener el movimiento indígena en Caldono dejó como el primer alcalde electo popularmente, en 1988, a Vitelio Meza, un indígena que se acompañó de cuatro líderes indígenas en el Concejo municipal.

En 1980 la violencia, resultado de la proliferación de distintos grupos armados, estalló en el país. Rappaport (2000) recuerda que las zonas rurales fueron atacadas duramente por el gobierno pues se consideraban "supuestos escondites de guerrilleros y campos de entrenamiento" (p. 176). La autora aclara cómo la constante persecución afectó la posibilidad de unificar un liderazgo político del movimiento indígena durante aquella década. El Cauca fue una de las principales zonas del país donde los distintos grupos guerrilleros tuvieron presencia permanente. Caldono no escapó a estas lógicas. Múltiples actores han pretendido su control político y militar: grupos paramilitares, fuerza pública y diversas insurgencias, entre las que se encuentran principalmente el Movimiento Armado Quintín Lame 2 , en la década de 1980, y las FARC-EP, en tiempos más recientes. Igualmente, por sus tierras también transitaron la Corriente de Renovación Socialista, M-19, y el Movimiento Ricardo Franco (facción disidente de las FARC).

Las FARC-EP hicieron presencia en la zona suroccidente del país de forma sostenida durante décadas. A partir de la Séptima Conferencia (1982), el grupo modificó sus formas de operar y privilegió las acciones militares ofensivas por sobre las tácticas de una guerra popular prolongada (Medina, 2011). En este sentido, el norte del Cauca se constituyó en un territorio de permanente enfrentamiento militar entre el Ejército y las

2 El Movimiento Armado Indígena Quintín Lame se constituyó en 1984 en el departamento del Cauca con el propósito de garantizar el respeto por la autonomía indígena, fortalecer y ampliar los territorios étnicos y manifestar el rechazo a la presencia de los demás grupos armados legales e ilegales en sus territorios. A finales de la década de 1980, este movimiento tuvo un apoyo significativo del grupo guerrillero M-19 tanto en el entrenamiento militar como en la formación política. Bajo la comandancia de Gildardo Fernández, el movimiento entró a hacer parte de la Coordinadora Guerrillera Simón Bolívar, que después se desmovilizaría (Guzmán y Rodríguez, 2015) y participaría en la asamblea constituyente. 
tropas de la guerrilla, así como con otros grupos armados ilegales como las Autodefensas Unidas de Colombia (AUC). Las AUC entraron al territorio a finales de la década de 1990 con el Bloque Calima, que tuvo el propósito de combatir el Frente 6 de las FARC-EP, ejercer control territorial y poblacional y concentrar el manejo de actividades vinculadas con el narcotráfico.

En el norte del departamento operaron el Frente 6, la Columna Gabriel Galvis y la Columna móvil Jacobo Arenas, que estuvieron bajo la coordinación del Bloque Occidental Comandante Alfonso Cano (BocAC). La presencia de las FARC-EP en el norte del Cauca estuvo estrechamente vinculada a su ubicación geográfica, pues representa un corredor estratégico que conecta el sur, norte y región Pacífico del país (Medina, 2011). Esta zona fue aprovechada no solo para el tránsito de soldados, sino también para el transporte de armamento, drogas ilícitas, suministros, secuestrados y el desplazamiento a zonas de retaguardia en momentos de enfrentamiento con la Fuerza Pública. Las condiciones de extrema pobreza y poca presencia estatal en las poblaciones rurales configuraron a la región como un espacio fértil para la formación de bases sociales y políticas de la insurgencia.

En el caso de Caldono, municipio donde se facilita la conexión y movilidad hacia el macizo colombiano y al departamento del Huila, la presencia y ofensivas militares entre las FARC-EP y la Fuerza Pública fueron constantes a través de hostigamientos y tomas de la cabecera. Según la investigación desarrollada por el Centro Nacional de Memoria Histórica y el Iepri, en Caldono se registraron treinta incursiones guerrilleras entre 1965 y 2013, dato que lo consolida como el segundo municipio, después de Toribío, en registrar mayor número de tomas en Colombia en ese periodo ${ }^{3}$. Al contrastar con otras fuentes, el CNMH-Iepri establece que en dicho periodo se presentaron además 37 hostigamientos en el

3 Según los datos del CNMH-IEPRI (2016), veintiocho de las incursiones se atribuyen a las FARC-EP, una al Movimiento Armado Quintín Lame y otro a un grupo guerrillero sin identificar. 
mismo municipio. En este sentido, se plantea que la presencia continua de las FARC en el corredor Toribío, Jambaló, Caldono, y más al sur, El Tambo, Argelia, Balboa, Bolívar, no solo pretende el despeje de la fuerza pública en la ruta de desplazamiento al Valle, del Cauca, Tolima y Huila, sino también "profundizar el influjo en zonas indígenas con la aspiración de adherirlas a su agenda política” (CNMH-Iepri, 2016, p. 71).

El reclutamiento y asesinato de comuneros y comuneras nasa a manos de las FARC-EP fueron motivo de pronunciamiento por parte de las comunidades indígenas, que permanentemente reivindicaron el control territorial a través de la guardia indígena ${ }^{4}$. Esta institución fue reconstituida en Caldono para responder a las emergencias; para el año 2000, contaba con 1000 guardias y en el corregimiento de Siberia llegó a incluir tanto a indígenas como a campesinos (CNMH-IEPRI, 2016).

En 1999 el alcalde de Caldono, junto a otros del departamento, fueron amenazados por las AUC, quienes los declararon objetivo militar debido a supuestos malos manejos de recursos públicos. La incursión paramilitar se acompañó de hostigamientos y asesinatos selectivos. Años después de su desmovilización, en 2011, las amenazas cayeron sobre indígenas misak y emisoras comunitarias nasa.

Los ataques en Caldono, dirigidos a la Policía ubicada en la cabecera, conllevaron la muerte de ocho policías, cinco soldados y cuatro ciudadanos, y dieciocho miembros de la fuerza pública y veintitrés civiles heridos, además de quince personas desaparecidas (CNMH-Iepri, 2016). Cabe resaltar que, el 12 de noviembre de 2001, las comunidades indígenas de este municipio protagonizaron un acto de resistencia civil pacífica que impidió la toma de la cabecera por parte de las FARC-EP. Según las fuentes consultadas por el CNMH-Iepri, se trató de una concentración de

4 Uno de los pronunciamientos provenientes de Caldono es el Acta de Andalucía, documento de 1985 en el que el CRIC agrupó varias denuncias de la interferencia de grupos armados, particularmente, las FARC-EP, en la cotidianidad de las comunidades indígenas del Cauca (Caviedes, 2007). 
unos 4500 indígenas en la plaza principal del poblado, que con quema de llantas, canciones y consignas obligaron a los insurgentes a retirarse.

Actualmente, en el departamento del Cauca se continúan reportando nuevas intimidaciones mediante panfletos que se atribuyen otros grupos paramilitares como las Águilas Negras e incluso las mismas AUC. Estas amenazas se dirigen a defensores y defensoras de los derechos humanos, pertenecientes a comunidades indígenas y campesinas y a movimientos sociopolíticos como Marcha Patriótica. Frente a este tipo de amenazas, se han activado alarmas por parte de distintas organizaciones para la defensa de los derechos humanos y las garantías de implementación de los acuerdos entre el gobierno y las FARC-EP.

El municipio también ha sido testigo de procesos de desmovilización en territorio indígena. El resguardo de Pueblo Nuevo fue el escenario para la concentración y desmovilización de 150 integrantes del Movimiento Armado Quintín Lame, quienes entregaron sus armas en este lugar en 1991. Además, Caldono fue paso transitorio para que los combatientes del M-19 se concentraran en zona rural de Toribío y, posteriormente, se desmovilizaran en 1990. Después de estar en el centro del conflicto armado y de procesos de negociación con otras guerrillas, los resguardos San Lorenzo de Caldono y Pueblo Nuevo decidieron en 2017 acoger el proceso de concentración y desarme de los excombatientes de las FARC-EP en su territorio, una vez firmado el Acuerdo Final para la Terminación del Conflicto y la Construcción de una Paz Estable y Duradera.

\section{La llegada de la Zona Veredal Transitoria de Normalización a Caldono}

El acuerdo de paz entre el Gobierno Nacional y la guerrilla de las FARCEP, firmado el 24 de noviembre de 2016, establece en el tercer punto, denominado "Fin del conflicto", la creación de las Zonas Veredales Transitorias de Normalización (ZVTN) y los Puntos Transitorios de Normalización (PTN), espacios definidos para que las y los excombatientes de esta insurgencia hicieran el proceso de dejación de armas y adelantaran los preparativos para su reincorporación económica, política y 
social a la vida civil. Se acordó, además, que el cese al fuego y la dejación de armas estarían vigilados por un mecanismo tripartito de monitoreo y verificación integrado por la Fuerza Pública, las FARC-EP y la misión de observación de la ONU.

Posterior a la firma del acuerdo final, el equipo negociador indicó que en La Habana se definieron algunos criterios para la localización de estos espacios, que restringieron su instalación en zona cocalera, de frontera, cabeceras municipales y territorios étnicos. Para entonces, se estableció una discusión entre el movimiento indígena y las FARC-EP, quienes indicaron que el criterio de ubicación por fuera de los territorios étnicos podía ser reevaluado, si las autoridades étnicas estaban de acuerdo.

A nivel nacional se establecieron diecinueve ZVTN y siete PTN, claramente delimitados territorialmente. Solamente en el departamento del Cauca se ubicaron dos ZVTN, en los municipios de Buenos Aires y Caldono, y un PTN, en Miranda. Desde su definición esos espacios serían de carácter temporal pues se guiaban por un cronograma consignado en el acuerdo final, que pretendía cumplir su objetivo en 180 días. Sin embargo, la logística y seguridad para el desplazamiento de excombatientes a estos espacios inició dos meses después de la fecha estipulada en el acuerdo, proceso que se dio sin que estos contaran aún con la infraestructura adecuada para su recibimiento.

La determinación de ubicar la ZVTN de Caldono en territorio étnico implicó procesos de discusión y tensión dentro del movimiento indígena a nivel nacional, en la Organización Nacional Indígena de Colombia (ONIC); departamental, en el Consejo Regional Indígena del Cauca; municipal, en la Asociación de Cabildos Indígenas de Caldono y la alcaldía; y local, en las asambleas de los cabildos de Pueblo Nuevo (Caldono-Silvia), San Lorenzo de Caldono, Tumburao (Silvia), Las Mercedes, Pioyá, La Aguada y La Laguna-Siberia. 
Para el año 2015, la discusión interna en los cabildos del municipio sobre su participación en la implementación del acuerdo de paz, aún en negociación, reconocía tres hechos fundamentales: 1) el impacto exacerbado del conflicto armado sobre las comunidades indígenas, reflejado, en parte, en la participación de comuneros y comuneras de los cabildos en las filas de actores armados; 2) la presencia de las FARCEP y de diversos grupos armados; y 3) las múltiples experiencias de desmovilización de otros tantos que han sucedido en sus territorios como aquella del Movimiento Armado Quintín Lame, la Corriente de Renovación Socialista, el M-19, el Movimiento Ricardo Franco (facción disidente de las FARC) y el JEGA (facción disidente del EPL).

En marzo de 2015, en asamblea comunitaria, Pueblo Nuevo definió "asumir el posacuerdo"; para tal fin, se introdujo en el plan de trabajo el desarrollo de espacios de reflexión y discusión sobre sus implicaciones, con el objetivo de concretar acciones a favor de la construcción de paz en el territorio. Para septiembre del mismo año, se estableció un acuerdo de cooperación con el Instituto de Estudios Interculturales (IEI) de la Pontificia Universidad Javeriana, en el que se proyectó "Pueblo Nuevo como una experiencia piloto de construcción de paz en el norte del Cauca”. A partir de esa fecha, se impulsaron procesos de socialización de los avances en los diálogos de La Habana, talleres de construcción de paz y planeación territorial, organizados por el instituto y por otras instituciones, con la participación de actores académicos y políticos que propiciaron el debate. A su vez, las autoridades indígenas del año 2015 y 2016 participaron en distintos conversatorios a nivel regional y nacional. Sin embargo, para ese momento ni en el país ni en la región había claridad sobre las definiciones tomadas por el equipo negociador en La Habana respecto al proceso de cese definitivo al fuego y de hostilidades.

Según la información recolectada en los procesos de acompañamiento del Instituto de Estudios Interculturales, los cabildantes del resguardo de Pueblo Nuevo rememoran dos antecedentes importantes para la toma de esta decisión. El primero es la definición de tres consignas por 
parte del movimiento indígena en la década de 1990, a saber: la necesidad de una salida negociada al conflicto, el ofrecimiento de su territorio para los diálogos de paz en aquel momento y la inclusión de la comunidad en una "conversa para construir el país en el que quepamos todos". El segundo antecedente es el XIV Congreso del Consejo Regional Indígena del Cauca - llevado a cabo en el Resguardo Indígena Kokonuko en 2013-, en el que se definió como prioritario el fortalecimiento interno de la organización indígena a través de los principios de unidad y autonomía. Por este motivo, las comunidades de base en las asambleas de los cabildos son las que toman las decisiones y las autoridades propias son quienes pueden comunicarlas. Para los cabildantes, estos antecedentes indican la coherencia de la decisión respecto a la ZVTN con los principios éticos y políticos fijados por el movimiento en relación con su papel activo en la construcción de paz; asimismo, reafirman su legitimidad, pues fue la asamblea del cabildo la que ratificó dicha decisión.

A inicios de 2016, los medios masivos de comunicación dieron a conocer a nivel nacional la figura de Zonas Veredales Transitorias de Normalización como las áreas de agrupamiento para la dejación de armas que estaba siendo pactada en La Habana. El punto sobre el "Fin del conflicto” del acuerdo entre las FARC-EP y el Gobierno Nacional no se firmaría hasta junio de ese año. La noticia de que una de estas ZVTN había sido proyectada por la guerrilla en la vereda Andalucía del resguardo San Lorenzo Caldono llegó por sorpresa a las autoridades de los cabildos y se difundió vía telefónica entre los resguardos.

Dos meses después, en septiembre, Pueblo Nuevo celebró una asamblea en la que convocó al Gobierno Nacional y de manera explícita indicó su deseo de acoger una de estas zonas en su territorio, acto que replicó el resguardo San Lorenzo de Caldono. Posteriormente, se inició la delimitación de predios y, en comunicación con el cabildo, se definieron los contratos de arrendamiento. 
Ahora bien, la instalación de la ZVTN no solo requería los predios para ambos campamentos que habitarían excombatientes de las FARC, también implicaba la instalación del lugar de operación del Mecanismo Tripartito de Monitoreo y Verificación. Según lo estipulado en el acuerdo, este mecanismo debía respetar el ancho de la zona de seguridad establecido en un kilómetro a la redonda de los campamentos, pues el monitoreo tuvo como matriz la separación de fuerzas entre el Ejército y la guerrilla. El mecanismo se instaló en la vereda La Venta en septiembre de 2016. La comunidad campesina interpretó inicialmente este alquiler como una oportunidad para aportar al proceso de construcción de paz y fortalecer económicamente la organización comunitaria. Sin embargo, meses después, en entrevistas con el IEI indicaron que la decisión se tomó aceleradamente y que los términos del contrato de alquiler convenidos de manera verbal no fueron cumplidos a cabalidad por parte de las instituciones en el momento de entrega del predio y finalización del contrato.

Finalmente, en diciembre de 2016, se inició la instalación de la Zona Veredal Transitoria de Normalización “Carlos Perdomo”, ubicada en la vereda San Antonio de los Monos en el municipio de Caldono y compuesta por dos campamentos en las veredas San Antonio y Santa Rosa, situados en territorio de los resguardos coloniales Pueblo Nuevo y San Lorenzo de Caldono. Allí se llevó a cabo el proceso de dejación de armas por parte de 510 excombatientes que pertenecían a la columna móvil Jacobo Arenas, cuya ocupación era principalmente la confrontación militar. Para esas fechas, las FARC-EP indicaban que la columna estaba conformada mayoritariamente por hombres, con un $82 \%$ en sus filas, frente a un $17 \%$ de mujeres. Del total de excombatientes allí concentrados, el $87 \%$ afirmaba pertenecer a una comunidad étnica. Estos datos rectifican que la afectación del conflicto armado en las comunidades indígenas del norte del Cauca conllevó un alto índice de reclutamiento de jóvenes en los resguardos, tal como lo denunciaron las autoridades tradicionales nasa, sin ignorar las incorporaciones voluntarias en dichas comunidades. 
Más recientemente, para mediados de 2017, la finalización del proceso de dejación de armas modificó varios de los esquemas establecidos en el municipio en el marco de la implementación del acuerdo. Por un lado, la primera misión de la ONU, cuyo objetivo era verificar el cese bilateral del fuego y hostilidades y acompañar el proceso de dejación de armas de la insurgencia, finalizó sus labores el 31 de agosto. La segunda misión entró en funcionamiento el 26 de septiembre, conformada únicamente por civiles, con el mandato de verificar el proceso de reintegración a la vida civil de los ahora excombatientes de las FARC. Por otro lado, los puestos de control militar, inicialmente establecidos en un perímetro de $3 \mathrm{~km}$ a la redonda de la zona campamentaria, tenían ahora la posibilidad de vigilar todo el territorio, lo que generó percepción de inseguridad por parte de las comunidades vecinas.

Finalmente, el Decreto 1274 de 2017 prorrogó las ZVTN y los PTN hasta agosto de ese año o hasta culminar el proceso de extracción de armas, después de lo cual pasaron a definirse como Espacios Territoriales de Capacitación y Reincorporación (ETCR). Según lo estipulado en el Decreto 2026 de 2017, en estos espacios se adelantarían actividades que facilitaran la reincorporación social y económica de las y los excombatientes de las FARC-EP, así como la implementación de sus proyectos productivos y el desarrollo de procesos de formación que vincularan de igual manera a comunidades aledañas. Esta transformación implica una permanencia más prolongada de exmiembros de esta guerrilla en territorio de los resguardos de Pueblo Nuevo y San Lorenzo de Caldono con miras a construir apuestas para reintegrarse a la vida civil.

Según datos de la Oficina del Alto Comisionado para la Paz, el censo poblacional en los ETCR muestra que en Caldono, al 15 de noviembre de 2017, permanecían 175 adultos, una cantidad significativamente inferior en comparación con la población concentrada inicialmente en la ZVTN. Esto puede explicarse, dada la alta pertenencia étnica de quienes allí se agruparon, quienes podrían haber regresado a sus lugares de origen en el mismo municipio o en cercanías. Igualmente, las condiciones 
de inseguridad por incursiones de otros actores armados en el territorio o los incumplimientos en lo pactado frente al acondicionamiento inicial de las ZVTN — que en Caldono presentó severos retrasos- pudieron haber favorecido el abandono del lugar. De cualquier manera, la instalación de la Zona Veredal en Caldono fue un proceso complejo que gestó tensiones en lo político entre las autoridades locales y algunos referentes de la organización indígena. Sin importar su denominación, esta situación continúa planteando preguntas alrededor de la reincorporación, la convivencia y el ejercicio de autonomía en el territorio.

\section{Tensiones en lo político: autonomía indígena y control territorial}

Cuando nos referimos a las tensiones en lo político y frente al proceso de reincorporación es fundamental diferenciarlas conceptualmente de los conflictos. En términos generales, el conflicto puede definirse como una situación de divergencia en la que se presenta una contraposición de intereses, necesidades y/o valores en pugna entre dos o más partes. En este sentido, los conflictos son consustanciales a las relaciones humanas e inevitables en la interacción social en todos los niveles, desde el interpersonal hasta el internacional y el étnico (Ruiz, 2005). Los conflictos surgen y se configuran en el marco de las relaciones de poder, por lo que su aparición apunta a un proceso social en el que “dos o más partes tratan de imponer valores excluyentes [...] a fin de influir en la conducta y el control de grupos sociales” (Pérez, 2003, p. 64).

En esta línea, Touzard (1981) planteó que los conflictos parecen responder al tipo de sociedad que los vive, por lo que manifiestan una particularidad respecto al momento histórico y al contexto de esta. Los conflictos cambian de acuerdo con las dinámicas sociales, económicas, culturales y políticas; de aquí que cada individuo o comunidad experimente y exprese los conflictos de manera diversa, que los asuntos contrapuestos varíen y que los intereses que mueven a estos actores se 
puedan modificar temporal y espacialmente. Igualmente, las formas de llevar los conflictos a una resolución son también diversas.

Las tensiones pueden entenderse como el germen de los conflictos, una etapa primaria que según las condiciones subsiguientes puede escalar a una confrontación con manifestaciones violentas o puede transformarse en una oportunidad de cambio que no recurra a repertorios de agresión. Bajo esta conceptualización, las situaciones observadas en Caldono son abordadas como tensiones que potencialmente pueden transformarse en conflictos territoriales. De acuerdo con Pérez, estos se originan "cuando el control y el dominio sobre espacios geográficos estratégicos se traducen en intereses de carácter político y económico" (2004, p. 65), es decir, aparecen en espacios que son valorados por su función productiva o que representan ventajas para el dominio de las administraciones locales y, podríamos agregar, de otros actores que busquen el control del territorio.

En este sentido, Caldono representa un espacio donde convergen los intereses de varias partes tanto por la presencia histórica de comunidades indígenas organizadas que poseen títulos coloniales sobre la tierra y han construido relaciones ancestrales de identidad y vida con su territorio como por su ubicación de corredor estratégico y zona de retaguardia para actores armados ilegales. La histórica presencia e incidencia del Sexto Frente de las FARC y de la Columna Móvil Jacobo Arenas, con una importante base de guerrilleros y guerrilleras de origen indígena de la zona del Cauca, aunada a la fuerte presencia de procesos milicianos en el territorio (Guzmán y Rodríguez, 2015), dejan entrever los fundamentos para que en este municipio coincidiera la propuesta de ZVTN con un territorio de reincorporación y de interés estratégico para el desarrollo del proyecto político y social de esta guerrilla.

Como se mencionó previamente, según los acercamientos del Instituto de Estudios Interculturales con las autoridades de los resguardos de Caldono, la ubicación de la zona veredal en su territorio causó tensiones en lo 
político que se remiten principalmente a cuestiones referentes a las instancias e instrumentos de gobierno propio y autonomía en el territorio. En primer lugar, se manifestó un disenso respecto a la decisión de ofrecer el territorio étnico para la instalación de la ZVTN entre las autoridades locales en el año 2016 y las organizaciones indígenas del nivel regional y nacional como ACIN, CRIC y ONIC. Los resguardos que apoyaron esta definición fueron Pueblo Nuevo, Tumburao (en Silvia) y San Lorenzo de Caldono. Por su parte, en 2016, el CRIC y la ONIC se pronunciaron públicamente mediante comunicados en contra de la instalación de zonas de concentración en territorios indígenas, ante lo cual exigían consulta previa frente al proceso de implementación del acuerdo de paz en estos lugares. Esta situación introdujo la discusión sobre el papel de las organizaciones de segundo nivel del movimiento indígena en los escenarios de toma de decisiones que competen a unos resguardos específicos. Los cabildantes de estos resguardos hicieron de forma insistente un llamado al reconocimiento de las asambleas de los resguardos y sus gobernantes (cabildantes) como autoridad tradicional encargada de ejercer y defender la autonomía indígena. A su vez, las organizaciones de segundo nivel son las que deben aconsejar y generar el debate político.

Tanto las dirigencias de estas organizaciones como los comuneros y comuneras del movimiento indígena temían en ese momento las afectaciones en términos de seguridad que podría representar la concentración de excombatientes de la guerrilla. El temor también contemplaba el posible impacto que la conformación del partido político Fuerza Alternativa Revolucionaria del Común pudiera tener en el movimiento indígena. Este partido era interpretado como una amenaza, ya que podría entrar en disputa por la elección de autoridades dentro de los cabildos, lo que se estableció como uno de los motivos por los cuales algunos de ellos no aprobaban la llegada de la ZVTN a territorio indígena. Algunos comuneros han denunciado que quienes apoyaron la decisión han sido estigmatizados como partidarios de las FARC: 
A nosotros nos montaron un cuento de que éramos disque de las FARC. Nos sacaron cuento de que éramos de la conpr [...] y, en el caso de Caldono, somos fieles a la dirigencia del CRIC. [...] El problema es que acá uno no puede decir cosas escandalosas porque de una te dicen que eres torcido o malo. Yo soy indígena, y soy censado, y uno no puede perder el punto de vista crítico. Para mí los conflictos son necesarios porque permiten ver los errores. (Líder indígena del municipio de Caldono)

Estos señalamientos tienen hondas repercusiones en un contexto como el departamento del Cauca, donde la presencia de grupos sucesores del paramilitarismo, así como también las amenazas y asesinatos a líderes y lideresas sociales van en aumento.

Las autoridades étnicas y la comunidad de los cabildos de Pueblo Nuevo y San Lorenzo de Caldono consideraron que la decisión de recibir la ZVTN en su territorio enviaba a la opinión pública un mensaje de respaldo y apoyo a la construcción de paz por parte del movimiento indígena. A su vez, reafirmaron la disposición de acoger a los comuneros y comuneras indígenas que permanecían alzados en armas, dada la reflexión de la pertenencia de una parte importante de ellos a las comunidades indígenas; incluso, analizaron como posible consecuencia la priorización de su territorio en el desarrollo de obras de infraestructura que solventaran las sentidas necesidades de la comunidad. En el debate, también se consideró la afectación, positiva o negativa, del movimiento indígena a partir de la puesta en marcha del partido político de las FARC y la dificultad que reviste el proceso de reconciliación en una zona que ha enfrentado las tragedias del conflicto armado.

Estos temores tienen correspondencia con las fricciones de larga data que se han presentado en las comunidades indígenas y los actores armados en el municipio de Caldono. Entre ellas, se pueden mencionar los reclamos de respeto hacia el territorio y los bienes sociales que han hecho los indígenas a las FARC en diversas ocasiones (CNMH-Iepri, 2016). Igualmente, "otro motivo recurrente de los roces con [esta 
guerrilla proviene] de que las autoridades indígenas le atribuyeran [...] la promoción del sembrado de cultivos ilícitos con el objetivo de lograr influencia en las comunidades y socavar el poder de las autoridades tradicionales” (p. 309), dinámica que se repitió por parte de grupos paramilitares en otras zonas del departamento.

Las tensiones políticas aquí mencionadas se remiten a cuestiones del ejercicio de autonomía y gobierno propio de los resguardos de Caldono, los cuales pueden verse disminuidos o potencializados por factores externos. En Colombia, a partir de la Constitución Política de 1991 y la legislación derivada, se reconoce a los resguardos indígenas como entidades territoriales con competencias administrativas, que cuentan con "estructuras políticas de autogobierno dentro de una jurisdicción legalmente reconocida [y un territorio delimitado] en donde ejercen derechos colectivos sobre la tierra y los recursos naturales" (Ulloa, 2010, p. 76). Para retomar a González, Ulloa (2010) comprende la autonomía como un mecanismo dispuesto por el Estado que propicia la autodeterminación de los pueblos indígenas respecto a sus derechos (étnicos y culturales) y, al mismo tiempo, permite mantener el orden estatal establecido. En palabras de esta autora, dentro de este sistema, el Estado colombiano reconoce formalmente la autonomía territorial y el autogobierno de los pueblos indígenas, lo cual se refleja en los niveles transnacional, nacional y local.

No obstante, Ulloa propone que la autonomía indígena en estos escenarios es un proceso complejo que se articula y se negocia con fuerzas externas de carácter local, regional, nacional y transnacional —según las circunstancias-. La autonomía, concluye la autora, "en esos contextos es un proceso de reconfiguración y confrontación frente a diversos actores” (p. 76). Esto se evidencia en los niveles mencionados, ya que en estos se introducen transformaciones en las dinámicas de los pueblos indígenas a partir de elementos como la transnacionalización de los recursos naturales en sus territorios, la incorporación fragmentada del enfoque étnico en las políticas gubernamentales y la presencia de 
actores exógenos en lo local que terminan por permear las acciones en territorios indígenas —como se argumentó anteriormente en relación con el conflicto armado en Caldono- - Todas estas dinámicas, en los diferentes niveles, inciden en la toma de decisiones autónomas por parte de los pueblos indígenas en sus territorios. Por ejemplo, Ulloa (2010) resalta la instalación de soberanías de hecho por parte de guerrillas y autodefensas en las instancias locales, en tanto que las manifestaciones violentas del conflicto han coartado la autonomía y el control territorial de las comunidades étnicas.

Para el caso de Caldono, la instalación de la ZVTN en los territorios étnicos permitió reconocer a las comunidades étnicas como actores fundamentales para la implementación exitosa del acuerdo; de esta manera, reconfiguró las dinámicas indígenas locales. Esta coyuntura puede generar alianzas para las comunidades indígenas de Caldono, pero también puede derivar, como señala Ulloa, "por un lado, [en] procesos de reconocimiento parcial e instrumental, y por otro lado, [en] una gobernabilidad parcial y limitada en sus territorios” (p. 78).

A esa gobernabilidad parcial y limitada hacen referencia las autoridades étnicas, cuando señalan otras tres tensiones evidentes. Una de ellas tiene que ver con la falta de canales de comunicación entre los actores que entran al territorio, en el marco de la implementación del acuerdo y las autoridades étnicas. Esto, según las comunidades indígenas, produce la marginación de la autoridad étnica en la toma de decisiones relacionadas con su territorio. Por este motivo, la comunidad propuso en distintas ocasiones la definición de un canal de comunicación directa entre estos actores externos y las autoridades indígenas y, en general, hizo un llamado al Gobierno Nacional para crear una ruta de coordinación que no se centrara exclusivamente en la administración municipal y departamental.

La siguiente tensión se asocia a las dificultades que la guardia indígena tuvo durante el primer año de funcionamiento de las ZVTN para 
garantizar el control territorial. Las guardias indígenas representan una extensión de las actividades que tradicionalmente han cumplido los alguaciles de los cabildos, encargados del control y la seguridad dentro de los resguardos. Están conformadas por comuneros y comuneras que son elegidas por las autoridades del cabildo y desempeñan acciones de vigilancia, control de la circulación, denuncia de irregularidades ante las autoridades comunales y llamados de alarma ante la presencia de grupos armados. En Caldono, la guardia ha llevado acciones de expulsión de armados en el territorio y de afirmación de la autonomía y la jurisdicción propia, de forma no armada. La tensión surge puntualmente frente a la inexistencia de canales de articulación de la guardia indígena de los cabildos Pueblo Nuevo y San Lorenzo de Caldono con el mecanismo tripartito de monitoreo y verificación de la zona; así como también, ante la negativa por parte del mecanismo a la solicitud del fortalecimiento de la guardia hecha por las autoridades étnicas, a propósito de lo inscrito en el capítulo étnico del acuerdo final:

Para el diseño e implementación del Programa de Seguridad y Protección para las comunidades y organizaciones en los territorios, se incorporará la perspectiva étnica y cultural. Se garantizará el fortalecimiento de los sistemas de seguridad propios de los pueblos étnicos, reconocidos a nivel nacional e internacional como la Guardia Indígena y la Guardia Cimarrona. (2016, p. 208)

Por último, el movimiento indígena, y en específico las autoridades étnicas del municipio, han ratificado su intención de impedirle a las instituciones nacionales e internacionales involucradas en la implementación del acuerdo imponerle una agenda al movimiento indígena que se limite a los compromisos allí consignados, pues esto desconocería la existencia de una agenda propia y el incumplimiento de acuerdos no asociados al proceso de paz pactados con entidades estatales que abordan asuntos que garantizan la gobernabilidad completa en sus territorios. 
La identificación de estas tensiones en el campo político indica que es fundamental el reconocimiento por parte de las instituciones públicas de los procesos autonómicos que las comunidades indígenas de Caldono mantienen en su territorio como el control territorial en cabeza de la guardia indígena, el reconocimiento interno de las autoridades tradicionales, la implementación de la jurisdicción especial indígena y el sistema de salud y educación propios, la recuperación de prácticas culturales ancestrales (entre estas, el Nasa Yuwe como lengua propia) y, en general, el desarrollo día a día de su plan de vida, su visión de futuro. Dicho reconocimiento implica la preparación de la institucionalidad para la comprensión de los vínculos de cooperación y articulación de estos proyectos propios de las políticas que se vienen estableciendo, a propósito de lo pactado en La Habana, de forma tal que la implementación del acuerdo fortalezca los procesos comunitarios sin coartar la autonomía. Con este objetivo, las instituciones deben establecer a las autoridades indígenas como principales interlocutores en la toma de cualquier decisión que implique a sus territorios.

\section{Compartir el territorio: la reincorporación de excombatientes}

Una segunda dimensión en la que se identificaron tensiones relevantes respecto a la instalación de la ZVTN en Caldono se relaciona con los asuntos que conciernen al proceso de reincorporación de exmiembros de las FARC-EP. Este proceso inicia con el cambio en el objetivo de la zona campamentaria gestada para la dejación de armamento, que posteriormente se configura como ETCR, espacio en el que se adelantarían las acciones necesarias para el reintegro de hombres y mujeres a la vida civil. Dichas tensiones se identificaron en tres ejes: el territorio y sus recursos, la reincorporación económica colectiva y la seguridad.

Las tensiones referentes al territorio se relacionaron con cuestiones sobre la tierra y el uso de recursos comunes, surgidas a raíz del hecho de tener que compartir el territorio con las y los excombatientes, actualmente concentrados en el ETCR. Así, los predios sobre los cuales 
se ubicaron los campamentos de excombatientes y el campamento del Mecanismo de Monitoreo y Verificación (MM\&V) en zona campesina presentaron dificultades con sus respectivos contratos de arrendamiento. En consecuencia, la comunidad indígena y campesina involucrada ha manifestado el desconocimiento o incumplimiento de los acuerdos inicialmente pactados con la institucionalidad. Particularmente, el campamento del $M M \& V$ fue instalado en una cancha comunitaria alquilada para tal fin por la comunidad campesina de la vereda La Venta. A finales de 2017, la comunidad señaló que el escenario sufrió deterioro y se tornó en un depósito de material de río y de las estructuras utilizadas para el campamento; este no fue restaurado por las instituciones competentes ni entregado formalmente a la comunidad en los tiempos pactados. Igualmente, durante su funcionamiento, no les fue permitido entrar al lugar en el que el MM\&V fue instalado ni participar de los espacios de diálogo, lo que generó la sensación de ser tratados como extraños en su propio territorio.

El uso del recurso hídrico también suscitó preocupaciones. El servicio de acueducto del resguardo de Pueblo Nuevo, que presenta condiciones precarias, fue compartido con las y los excombatientes. Esta situación conllevó la racionalización del consumo de agua para cubrir la totalidad del resguardo y dio lugar a una tensión que ha ido desapareciendo, pues para finales del año 2017 el número de excombatientes que habitaban el ETCR disminuyó sustancialmente. Los cabildantes reportaron que, aproximadamente ochenta personas permanecían diariamente en el campamento. De igual modo, existe una preocupación por parte de comuneros y las autoridades del resguardo en cuanto al déficit de tierra que se presenta, pues se teme que los proyectos productivos de las y los excombatientes requieran el uso de tierras pertenecientes al resguardo, ya que el nivel de hacinamiento es alto, incluso antes de iniciar el proceso de reincorporación.

Asimismo, ha surgido la pregunta en torno a la futura dedicación de la infraestructura del ETCR una vez las y los excombatientes abandonen el lugar. Las autoridades del resguardo han ofrecido varias propuestas de utilización del espacio que presentaron a las instituciones estatales 
competentes $^{5}$, pero siguen sin obtener respuesta. Entre estas, se encuentra la habilitación de programas de educación superior en dichas instalaciones y la construcción de un centro de investigación agropecuaria que permita impulsar la diversificación y tecnificación de cultivos. Dichas propuestas buscan solventar necesidades en lo social y productivo, que no han sido atendidas por la institución pública ni privada.

En razón a la expectativa sobre el proceso de reincorporación económica colectiva, se identificaron situaciones potencialmente conflictivas relacionadas con posibles escenarios de competencia entre las propuestas productivas de las comunidades indígenas y campesinas y la organización nacional Economías Sociales del Común (Ecomun) ${ }^{6}$. Ante esto, las autoridades de San Lorenzo de Caldono y Pueblo Nuevo han expresado su apertura al diálogo con los responsables del proceso de conformación de Ecomun en el municipio, de manera que se conozcan sus iniciativas y se logre generar trabajo conjunto. El cabildo de Pueblo Nuevo, en particular, ha propuesto que la cooperativa se enfoque en el desarrollo de actividades que resuelvan los problemas de comercialización y transformación de los productos agrícolas de las familias indígenas y campesinas del sector como los frutales de clima frío, café y fibra de fique. La cooperativa, por su parte, ha diseñado cuatro proyectos productivos agropecuarios y ha convenido con el resguardo de Tumburao (municipio de Silvia) el uso de un predio (a través de un comodato) que se destinará a la siembra de aguacate y de algunos cultivos transitorios. Ahora bien, los distintos actores con propuestas productivas coinciden

5 Oficina del Alto comisionado para la Paz (OACP) y Agencia para la Reincorporación y Normalización (ARN).

6 Cooperativa que surge de lo pactado en el acuerdo de paz como forma de promover el proceso de reincorporación económica colectiva. Su lanzamiento oficial se hizo en julio de 2017 y desde entonces las personas en proceso de reincorporación en el municipio de Caldono iniciaron su proceso desde lo local. Allí nació la Cooperativa Multiactiva Ecomun Esperanza del Pueblo (Coomeep), que a inicios del año 2018 contaba con cuatro proyectos productivos diseñados, en proceso de aprobación por el Consejo Nacional de Reincorporación, instancia encargada de validar su viabilidad para destinar posteriormente el presupuesto convenido en el acuerdo final. 
en la necesidad prominente de dotar al municipio con la infraestructura productiva idónea para la sostenibilidad de los proyectos que garanticen el éxito de la reincorporación. De igual forma, exponen su crítica a las instituciones que hacen presencia en la zona por el retraso evidente en los tiempos para el inicio de estos proyectos productivos.

Otro aspecto que ha generado dudas sobre la reincorporación se relaciona con la seguridad en el territorio; la presencia de actores armados ilegales es una situación que se agravó después de la firma del acuerdo de paz y que ha levantado las alarmas a nivel nacional. Desde inicios de 2017, las autoridades de los seis cabildos de Caldono denunciaron la ocurrencia sistemática de hechos violentos en sus territorios, como la amenaza, asesinato y desplazamiento de comuneros, guardias, dinamizadores, autoridades tradicionales, defensores y defensoras de los derechos humanos y la población en general, por parte de grupos armados autoidentificados como Águilas Negras y Ejército de Liberación Nacional, así como de otros no identificados. Igualmente, han sido objeto de restricciones al libre tránsito en el territorio en horas de la noche y de reclutamiento de jóvenes por parte de grupos paramilitares, quienes ofrecen remuneración y han instalado recompensas por las autoridades tradicionales. Se denunciaron, además, amenazas, chantajes y extorsiones de parte de dichos grupos a comerciantes y población de otros sectores del municipio. Finalmente, también se mencionó el nacimiento de una disidencia de las FARC que, hasta la fecha, no ha tenido encuentro alguno con las autoridades étnicas.

A su vez, la percepción general de seguridad en el municipio, expresada por pobladores cercanos a las zonas campamentarias, apuntaba a que, posterior a la concentración de excombatientes en los puntos de preagrupamiento, aumentaron los delitos menores y la delincuencia común. Según las comunidades indígenas y campesinas, esta situación no ha sido atendida por la Policía Nacional ni por la alcaldía municipal, lo que genera un clima de zozobra en la población que tiende a asociarse con la llegada de miembros de FARC al territorio para su proceso de dejación de armas. 
Un tercer actor que ha influido en la percepción de inseguridad en las poblaciones cercanas a los campamentos son las fuerzas militares, que se instalaron como parte del MM\&V y de los anillos de seguridad para la concentración en la ZVTN. Como se dijo anteriormente, con los Espacios Territoriales para la Capacitación y Reincorporación se dispuso la posibilidad de vigilancia militar sobre todo el territorio, en contraste con los anteriores puestos de control militar establecidos en un perímetro de $1 \mathrm{~km}$ a la redonda de la zona campamentaria. Esta situación provocó una tensión evidente en la comunidad indígena, que históricamente ha expulsado de su territorio a todos los actores armados, tanto legales como ilegales. Algunos pobladores indígenas de la zona señalaron que la presencia del cuerpo militar afectaba el proyecto de vida de los y las jóvenes de los resguardos, puesto que la carrera militar se presentaba como una opción de desarrollo personal que está en contra de los ideales pacifistas y antimilitaristas del movimiento indígena. A su vez, algunas personas se han sentido confinadas en sus predios, producto del control militar del Ejército Nacional.

\section{Reflexiones finales}

El municipio de Caldono es un territorio que mantiene las huellas ancestrales del pueblo nasa. Fuertemente custodiado por las comunidades indígenas, logró resistir la invasión española por un siglo, para luego formar parte del cacicazgo de Pitayó en cabeza de Juan Tama de la Estrella, con lo cual, se aseguró ciertas formas de autonomía y autogobierno durante la Colonia. Su historia lo ha conservado como territorio principalmente indígena, donde la presencia campesina se concentra en la zona baja con mayor acceso a carreteras para la comercialización de productos agropecuarios. Los fuertes procesos de despojo de tierras colectivas para privilegiar la titulación individual en la primera mitad del siglo XIX, que resultaron en la disolución del resguardo La Aguada, impulsaron con ímpetu su posterior recuperación al punto de consolidar la mayor parte de la zona alta del municipio como resguardo en lo que otrora fuera la tierra del cacique Juan Tama. 
La recuperación de terrenos en las décadas de los sesenta y setenta se cimentó en procesos de fortalecimiento importantes como la creación de la ANUC y, posteriormente, del CRIC. Actualmente, los seis cabildos del municipio presentan sistemas consolidados de autogobierno, que logran interlocutar con las instituciones de los niveles local, regional y nacional. Igualmente, son sujetos con un importante grado de articulación política y administrativa, sobre la base de un territorio ancestral compartido, apropiado y reivindicado.

La pervivencia de los conflictos configura las dinámicas territoriales en este municipio. Por un lado, se presentan disputas de tierras entre el pueblo misak y nasa en cercanías al resguardo La Laguna-Siberia, que se remontan a sus enfrentamientos precolombinos, ahondados durante la Colonia. Estos desencuentros se fundamentan en el saqueo inicial de las tierras por parte de los españoles y la posterior parcelación y disolución de los resguardos llevada a cabo por los colonos. Un actuar desinformado del Gobierno ha contribuido a que estos conflictos se profundicen y causen serias afectaciones a ambos pueblos.

Así mismo, la presencia histórica de actores armados alimenta la particularidad del municipio. Las autoridades indígenas y los comuneros y comuneras fortalecidas han hecho frente a las ocupaciones de grupos armados insurgentes y han afirmado su autonomía a través de acciones como la consolidación de la guardia indígena, actos de resistencia civil y, en otros niveles, el ejercicio de su sistema de justicia y educación propia.

En este contexto, el ofrecimiento del territorio indígena para la instalación de una ZVTN significó una apuesta contundente por la construcción de paz en el país, sin desconocer las tensiones que podría acarrear. La comunidad nasa de Caldono pone en evidencia un ejemplo de resistencia frente a las adversidades del conflicto: primero, por la ocupación de la Corona española; segundo, por mantener un territorio que logró conservarse al ser designado como resguardo; y ahora, por defenderlo de incursiones armadas y de la explotación económica y ambiental que puede 
avecinarse. Para retomar la idea de la bipolaridad del poder planteada por Rojas (1993) como eje de este municipio, cabe decir que el polo indígena ha resistido no solo a las lógicas clienterales y al poder estatal, como el autor establece, sino más recientemente al control territorial de actores armados, con quienes se han generado fricciones, acercamientos y desencuentros y ahora se apuesta por una convivencia conjunta.

A lo largo del primer año de implementación del acuerdo de paz, las comunidades indígenas de Caldono reconocieron una gran oportunidad para la reconciliación y el fortalecimiento territorial en la adopción de una ZVTN, con el propósito de garantizar el desarme y la reintegración a la vida civil de los y las excombatientes de las FARC. Con este gesto, han enviado un mensaje político al movimiento indígena y a la comunidad nacional e internacional, al apostarle a la construcción de paz desde lo local. Después de instalada la ZVTN, se han presenciado innumerables gestos de reconciliación en lo simbólico y lo cotidiano. Por ejemplo, se establecieron procesos de formación de conjuntos entre comunidad y excombatientes, como la validación del bachillerato en el ETCR, a la que se unieron comuneros del resguardo, y el recibimiento de quince niños y niñas hijas de excombatientes como estudiantes en la escuela de Pueblo Nuevo. También, se produjo un acuerdo entre un resguardo indígena y la cooperativa Ecomun del nivel municipal, para la utilización de un predio en uno de los proyectos productivos diseñados para la reincorporación, con lo cual se puede superar uno de los obstáculos más importantes en el proceso de desarrollo de iniciativas productivas de las FARC. Igualmente, se celebraron con frecuencia torneos de fútbol, en los que se encontraron habitantes del resguardo y excombatientes, quienes también han desarrollado tareas comunitarias para mejorar bienes compartidos como la carretera o el acueducto.

Pero quizás uno de los más destacables fue el acto de recibimiento por parte de las autoridades étnicas a los excombatientes indígenas como comuneros de los resguardos del municipio. Este acto se celebró a finales de 2017, inició con un ejercicio de armonización y finalizó con la 
socialización de las normas de convivencia que día a día deben cumplir todos las y los comuneros. Estos ejemplos de reconciliación indican que los procesos emprendidos por las comunidades indígenas desde décadas atrás se posicionan como un apoyo importante para la reincorporación de excombatientes en el municipio, situación que se hace posible gracias al gran número de personas pertenecientes a resguardos en las filas del movimiento insurgente.

En un territorio como Caldono, donde conviven actores variados con intereses distintos, la convivencia conjunta solo podría plantearse desde una perspectiva intercultural. Desde la filosofía política, la interculturalidad surge como respuesta conceptual y política al multiculturalismo, el cual busca reconocer y preservar la diferencia social, bien sea en términos éticos o diferenciales (Kimlicka, 1996; Touraine, 1997). La interculturalidad integra el principio de la interacción positiva de la diversidad cultural y reconoce lo difuso de las fronteras culturales en sociedades que comparten territorios. Así, este concepto propone la construcción de un espacio para interlocutar con la otredad, sea cual sea su forma. Aunque en su propuesta desde los movimientos sociales ha tenido diversos abordajes, hablar de interculturalidad permite plantear preguntas de fondo sobre la jerarquía y la simetría de la organización social para pensar en una transformación estructural que sea posible mediante un diálogo transformador. De igual manera, esta señala hondos cuestionamientos en relación con el sistema económico y político liberal y las "concepciones monoculturales y excluyentes" del multiculturalismo estatal (Walsh, 2002). En este sentido, un proceso de diálogo intercultural que pretenda acercar las posturas opuestas de los actores implica el reconocimiento de la polifonía de voces que se ciernen sobre el territorio, de manera que pueda acercar esas distintas visiones sin la intención de eliminarlas o integrarlas para construir puntos en común.

A lo largo del trabajo realizado por el Instituto de Estudios Interculturales con diferentes actores en contextos rurales, se ha encontrado que este proceso resulta en un aprendizaje mutuo, gracias al cual surgen 
posibilidades para la convivencia, la cooperación en objetivos y los proyectos comunes. Esto deriva del cuestionamiento de las posturas propias de cada parte en diálogo, así como de la apertura para construir confianza y consensos. Para el caso de las tensiones surgidas en los resguardos de Pueblo Nuevo y San Lorenzo de Caldono por la instalación del actual ETCR en su territorio, resulta necesario fortalecer el enfoque étnico planteado en el acuerdo de paz y aplicarlo desde una perspectiva de diálogo intercultural. Esto contribuye a consensuar las maneras más apropiadas para aplicar los lineamientos del capítulo étnico, según la especificidad de un territorio tan complejo como el aquí descrito.

De este modo, aspectos como el fortalecimiento y articulación de la guardia indígena frente al ejercicio de control territorial y vigilancia del ETCR, la coordinación de iniciativas productivas de Ecomun con proyectos en marcha de los cabildos y las directrices de comunicación con instituciones públicas y privadas que llegaron a intervenir en el territorio, pueden fortalecerse si los actores involucrados se comprometen en un proceso auténtico de construcción de consensos y logran entender y respetar sus diferencias y coincidencias desde lo político, lo económico e incluso lo cosmogónico.

Ahora bien, la necesidad de generación de consensos frente a las particularidades en la implementación del acuerdo final en este territorio étnico sigue siendo vigente. Actualmente, no solo compete a las autoridades étnicas, a las FARC y a las instituciones públicas que desarrollan las políticas convenidas en La Habana, sino también a todas las organizaciones de la sociedad civil interesadas en generar apoyos, acompañamientos o intervenciones en pro de la construcción de paz en el municipio.

Finalmente, esta propuesta no es ingenua respecto a las tensiones que surgen en el encuentro con el otro, puesto que, como se mencionó antes, el conflicto es inherente a las relaciones humanas. Se reconoce entonces que en el centro de la convivencia y el diálogo están los 
conflictos entre quienes comparten un mismo territorio. Los procesos de reincorporación y reconciliación, en tanto se basan en la convivencia de diferentes, deben considerar la construcción de diálogos y rutas participativas en las que se alcance un consenso en torno a las intervenciones futuras, especialmente aquellas proyectadas entre actores con cosmovisiones heterogéneas.

\section{Bibliografía}

Bonilla, Víctor Daniel (2012). Resistencia y luchas en la memoria misak. En C. N. Histórica, Nuestra vida ha sido nuestra lucha, pp. 121-165. Colombia: Taurus, Semana y Centro de Memoria Histórica.

Caviedes, Mauricio (2007). Guardias indígenas de la zona centro y Caldono. En M. Caviedes (Ed.), Paz y resistencia: experiencias indígenas desde la autonomía, pp. 56-78. Bogotá: Cecoin.

Centro Nacional de Memoria Histórica y el Instituto de Estudios Políticos y Relaciones Internacionales (2016). Tomas y ataques guerrilleros (1965-2013). Bogotá: Centro Nacional de Memoria Histórica.

Departamento Administrativo Nacional de Estadística (2005). Estimación y proyección de población nacional, departamental y municipal por sexo, grupos quinquenales de edad y edades simples de 0 a 26 años 1985-2020. Recuperado de: https://bit.ly/2slBjnd

Departamento Administrativo Nacional de Estadística (2014). Censo Nacional Agropecuario. Recuperado de: https://bit.ly/2D9AlBa

Guzmán, Álvaro y Rodríguez, Alba Nubia (2015). Orden social y conflicto armado. El norte del Cauca 1990-2010. Cali, Colombia: Universidad del Valle.

Kymlicka, Will (1996). Ciudadanía multicultural. Una teoría liberal de los derechos de las minorías. Barcelona: Editorial Paidós.

Medina Gallego, Carlos (2011). FARC-EP. Flujos y reflujos. La guerra en las regiones. Bogotá: Universidad Nacional de Colombia. Facultad de Derecho, Ciencias Políticas y Sociales. Instituto Unidad de Investigaciones JurídicoSociales Gerardo Molina. 
Oficina del Alto Comisionado para la Paz (2016). Acuerdo Final para la Terminación del Conflicto y la Construcción de una Paz Estable y Duradera. Recuperado de: https://bit.ly/2mXa6W6

Oficina del Alto Comisionado para la Paz (2017). Espacios territoriales de capacitación y reincorporación. Vereda San Antonio de los Monos. Bogotá. Recuperado de: https://bit.ly/2087VZe

Pérez Martínez, Manuel Enrique (2003). La conformación territorial en Colombia: entre el conflicto, el desarrollo y el destierro. Cuadernos de Desarrollo Rural, (51), pp. 61-90. Bogotá: Pontificia Universidad Javeriana.

Rappaport, Joanne (2000). La política de la memoria. Interpretación indígena de la historia en los andes colombianos. Popayán: Editorial Universidad del Cauca.

Rojas Guerra, José María (1993). La bipolaridad del poder local. Caldono en el Cauca indígena. Cali: Universidad del Valle.

Ruíz, Manuel Ángel (2002). Visiones de la Etnicidad. Revista Reis, (98), 79-106.

Touraine, Alain (1996). ¿Podremos vivir juntos?: iguales y diferentes. Argentina: Fondo de Cultura Económica.

Touzard, Hubert (1981). La mediación y la solución de los conflictos. Barcelona, España: Herder.

Ulloa, Astrid (2010). Reconfiguraciones conceptuales, políticas y territoriales en las demandas de la autonomía de los pueblos indígenas en Colombia. Tabula Rasa, (13), 73-92. Bogotá.

Walsh, Catherine (2002) (De)construir la interculturalidad: consideraciones críticas desde la política, la colonialidad y los movimientos indígenas y negros en el Ecuador. En N. Fuller (Ed.), Interculturalidad y política: desafíos y posibilidades, (pp. 115-142). Lima: Pontificia Universidad Católica del Perú, Universidad del Pacífico, Instituto de Estudios Peruanos (IEP). 